Article

\title{
Living Systems Escape Solipsism by Inverse Causality to Manage the Probability Distribution of Events
}

\author{
Toshiyuki Nakajima
}

check for

updates

Citation: Nakajima, T. Living Systems Escape Solipsism by Inverse Causality to Manage the Probability Distribution of Events. Philosophies 2021, 6, 11. https://doi.org/10.3390/ philosophies6010011

Received: 31 December 2020

Accepted: 4 February 2021

Published: 9 February 2021

Publisher's Note: MDPI stays neutral with regard to jurisdictional claims in published maps and institutional affiliations.

Copyright: (C) 2021 by the author. Licensee MDPI, Basel, Switzerland. This article is an open access article distributed under the terms and conditions of the Creative Commons Attribution (CC BY) license (https:// creativecommons.org/licenses/by/ $4.0 /)$.
Department of Biology, Ehime University, Matsuyama, Ehime 790-8577, Japan; cognizers@yahoo.co.jp

\begin{abstract}
The external worlds do not objectively exist for living systems because these worlds are unknown from within systems. How can they escape solipsism to survive and reproduce as open systems? Living systems must construct their hypothetical models of external entities in the form of their internal structures to determine how to change states (i.e., sense and act) appropriately to achieve a favorable probability distribution of the events they experience. The model construction involves the generation of symbols referring to external entities. This paper attempts to provide a new view that living systems are an inverse-causality operator. Inverse causality (IC) is an algorithmic process that generates symbols referring to external reality states based on a given data sequence. For applying this logical model involving if-then entailments to living systems involving material interactions, the cognizers-system model was employed to represent the IC process; here, living systems were modeled as a subject of cognition and action. A focal subject system is described as a cognizer composed of sub-cognizers, such as a sensor, a signal transducer, and an effector. Analysis using this model proposes that living systems invented the "measurers" for conducting IC operations through their evolution.
\end{abstract}

Keywords: living systems; solipsism; inverse causality; external reality; probability; measurement; measurer; cognizer

\section{Introduction}

Living systems need to survive and reproduce in their uncertain environments. This representation is the externalist view of living systems, i.e., seeing living systems and their environments as objects from an external observer, such as a biologist. This viewpoint is mainstream in science.

In contrast, the internalist approach attempts to understand a system from within. Here, we define this approach as a methodology to understand living systems without assuming their external world. In this view, we have a different stance from the above statement: (i) The external world or reality does not objectively exist because it is unknown by any living system from within. Instead, living systems each construct their own specific models of hypothetical external entities in the form of their internal structures that determine how to change states. (ii) Based on their models, they change their states (i.e., sense and act) appropriately to achieve a favorable probability distribution of the events that they experience [1-5]. The model construction by living systems involves the generation of symbols for external entities (e.g., producing second messenger molecules or neural activity patterns) and functions to process them in their specific way for survival and reproduction. As a result, the probability distribution of events that each living system will experience forms a subject-based environment, called Umwelt [6,7].

Internally-constructed environment models may vary according to systems, even under an identical environment recognized by an external observer. Therefore, exploring the internal model construction is an essential part of understanding how each system adapts to the environment. The construction of the external reality model is regarded as an adaptation to behave in an adaptive way to the environment, not as the truth 
about its external reality [8]. Living systems behave using perceptual objects or signals generated within, referring to external entities unconfirmed directly. The subjective activity of generating them varies according to systems that differ on how to survive and reproduce. The internalist stance illuminates how living systems construct their specific models for their hypothetical external worlds based solely on the phenomena (events, percepts, or data) they each have.

The internalist approach involves a philosophical issue concerning the relationship between "I" (the self or mind) and the external reality (world). Descartes' skepticism uncovered the distinction between percepts (cogito) and the objects' reality to which the percepts refer. Therefore, the semantic content (e.g., an image of "this apple") cannot provide a secure base for confirming the existence of such reality. Husserl's phenomenology attempts to clarify how a mental process can generate perceptual objects for things that may exist outside (i.e., transcendent being). He highlights intentionality, i.e., the directedness or aboutness of consciousness, as playing a pivotal role in singling out a particular object in addition to the importance of the intersubjective constitution $[9,10]$.

Despite Husserl and his followers' efforts, it remains unclear how a mental process can distinguish between perceptual objects in dreams or hallucinations and those in real life [11-13]. Maturana maintained the indistinguishability in the operation of the nervous system between perception and hallucination: "perception should not be viewed as a grasping of an external reality, but rather as the specification of one, because no distinction was possible between perception and hallucination in the operation of the nervous system as a closed network" [14] (Introduction).

However, living systems, such as cells and organisms, are not closed systems from an externalist view. They are open in their metabolic processes, requiring resources from outside and discarding waste metabolites outside. Suppose that living systems can have no criterion by which they distinguish between perception relating to the external reality and hallucination (perception relating to internal process). How can living systems maintain their structure as open systems? If an animal is subject to hallucinations, it will not survive long. Even bacteria have this problem. If indistinguishability is valid in living systems, it could limit the internalist approach to exploring living systems.

The difficulty in solving the problem of the percept-reality relationship partly originates from a circular relationship between phenomena (data) and external reality, an enduring fundamental issue in philosophy and science $[6,7,9,10,14-21]$. The external reality produces (or causes) phenomena (data) by observation or measurement. In contrast, the phenomena provide evidence to confirm the reality. We cannot determine whether an observed object produces (causes) data or the data produce (derive) the object [21]. (The former view includes the idea that the interaction between the subject and the reality produces data, which is an externalist view that admits the subject and the external entities both as objects.)

The former sense of data is the ordinary type of information, such as Shannon's information: a message sent from a person to a receiver [22]. The latter sense of data, those creating the object, is the it-from-bit type of information, originating from quantum physics [17]. It-from-bit says that every "it" (i.e., any physical reality, including the spacetime continuum) derives its function, meaning, and very existence entirely from the apparatus-elicited answer to yes or no questions, bits. The internalist stance we take in this paper concerns the it-from-bit type of data or information. However, that problem arises again as to how can "it" be distinguished from a hallucination.

A promising strategy for solving the percept-reality problem focuses on the syntactic structure of percepts (data) rather than on their semantics. Nakajima [23] termed the generation of symbols for external realities as "realization", i.e., making it real from phenomena (data), to distinguish it from "observation" (or "measurement") by which the external reality produces phenomena. The construction of an external-reality model within a subject (i.e., realization) is searching for a way to escape solipsism. A study [23] provided a simple model to approach the problem. 
A sequence of meaningless symbols is given, which is then processed by a principlebased algorithmic operation, called inverse causality (IC), to generate symbols that do not belong to the sequence. The IC model considers that the generated symbols refer to states of the external reality or entity, undifferentiated into various entities (reviewed in Section 2). The study also revealed that the IC process occurring within the self (subject) is equivalent to the measurement of the external object when viewed from the external observer (Section 3). In other words, IC and measurement can be different aspects of the same thing. This equivalence can be a key to integrating the internalist and the externalist frameworks toward a comprehensive framework of understanding living systems.

However, we still have a problem to solve. The IC model is a logical (epistemic) model, not a process proceeding in a spatial-temporal space. Therefore, it is required to convert logical entailments (if-then) in the IC model into an externalist model, i.e., a material-system model, to make the IC process applicable to living systems.

With this aim, in this paper, we attempt to transform the IC model into the cognizerssystem model that describes material entities, such as living systems, as a subject of cognition and action [23,24]. Next, we describe a focal subject as a cognizer composed of sub-cognizers, such as a sensor, a signal transducer, and an effector; then, we explore how the subject system can generate symbols for the external reality or entities using the IC operation; and, finally, we discuss how this symbol generation can link to effector actions to affect the probability distribution of events that a focal living system experiences.

\section{The Inverse Causality Model: Overview}

Aiming to solve this puzzling problem, Nakajima [23] provided a simple mathematical scheme by transforming the problem into a tractable form. First, standing upon a solipsistic stage, "the self" is defined as the entire spectrum of semantic, perceptual contents generated by mental activity, involving sensation, cognition, ideation, will, imagination, feeling, and thought (Figure 1a). Each specific content is called a perceptual element, distinguished from one another by the self. This spectrum covers the whole world, which is a solipsistic world [23] (p. 685). This departure point is almost the same as the one that Husserl took in his phenomenological study, in which he considers "mental processes of consciousness in the entire fullness of the concreteness within which they present themselves in their concrete context sense is changed to range entirely the stream of mental processes" [9].

Next in the scheme, the self is abstracted into a particular finite sequence of perceptual elements (designated $M$ with the set of the elements $\mathbf{M}$ ), written as a sequence of symbols. Different symbols indicate different percepts distinguished from others by the self (Figure 1b). The sequence is a chronicle of perceptual elements arrayed in a time series. Each element cannot be said to be caused or entailed by its predecessor. That is, it is given as it is, as a direct experience for the self.

Here, the semantic contents of perceptual elements are distilled out, and only their order in time and distinctness is identified. This sequence is the complete wholeness, as in solipsism, assumed at the departure point of the IC model. $M$ is the first-order reality in this sense. Without it, nothing can happen. Here, the outer realm for the self can only exist as the semantics of symbols formed within $M$. However, it turned out that $M$ is not identical with the self. The self includes a larger domain of perceptual sequences and the properties to process $M$, as is evident in the following argument (see also [23] (p. 695)).

Then, we can ask: How can the self operate a given perceptual sequence to generate (derive) new symbols that do not belong to the set $\mathbf{M}$ ? An answer to this question is an algorithmic operation called inverse causality, denoted "IC". IC can generate new symbols from particular structures of a sequence based on the unique successor principle (Figure 1c). Given a sequence, such as $m 1, m 3, \ldots, m 1, m 7$, the $m 1$ does not have a unique successor. Then, new symbols $e 0^{*}$ and $e 1^{*}$ are introduced behind the first and second $m 1$, respectively, to disambiguate the difference. 
(a)

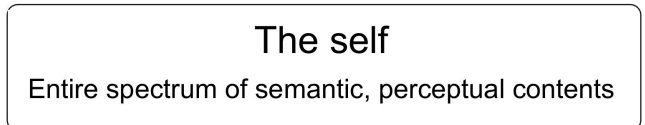

(b)

Temporal sequence of meaningless symbols $M$ $m 1, m 3, m 3, m 8, m 1, m 7, \ldots$

(c)

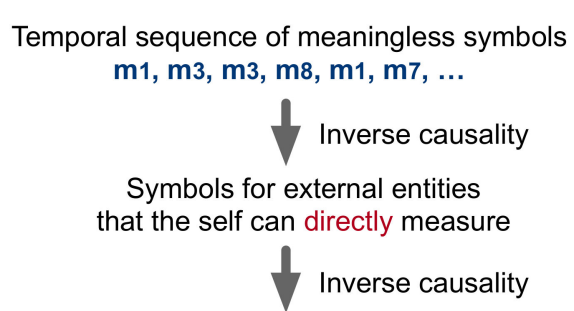

Symbols for external entities that the self can indirectly measure

Figure 1. Overview of the previous study [23]. (a) The self is hypothetically modeled as an entire spectrum of percepts, which are not entailed (or caused) by something but given as they are. (b) The self is abstracted as a temporal sequence of meaningless symbols, which are given as they are. (c) New symbols referring to external entities are derived (entailed) from the meaningless sequence by the algorithmic operation of inverse causality.

The IC process can be seen as if the self does measure an external entity when the operation is viewed in the reversed direction: the self in state $m 0$ measures the external entity $E$ in state $e 0$ (or $e 1$ ), changing its state to $m 3$ (or $m 7$ ). Here, symbols $e 0^{*}$ (or $e 1^{*}$ ) are generated within the self, which refers to the unknown external reality $E$ in $e 0$ (or $e 1$ ) with the state set $\mathbf{E}$. The subject cannot directly experience $E$, but can only generate symbols, $e 0^{*}$ and $e 1^{*}$, internally that refer to $E^{\prime}$ s states. The IC operates on a given sequence $M$ (not entailed by something) to generate a new sequence, including elements referring to an external reality that can be directly measured; this operation is denoted ICM.

For an external observer, the IC process is perceived as a process in which an external entity causes (produces) the data used for the symbol generation by the subject. Note that IC is not a process in which an event-as-effect generates an event-as-cause.

IC operates again on the entire sequence, including derived symbols, which can derive new symbols (Section 3.3). The operation on the whole level of sequence, therefore called ICW, generates a new sequence, including elements referring to an external reality that can be indirectly measured.

The IC model identifies the external reality as the set of states that do not belong to the given sequence $M$, whose elements (states) the IC-derived symbols refer to. The reality is a single entity and not differentiated into a plural number of entities. Due to this undifferentiated status, the reality is called the proto-reality [23].

The previous model [23] describes that the IC-derived elements are formed downstream of an $M$ sequence (p. 695). However, the word "downstream" is confusing because, by definition, $\mathbf{M}$ cannot contain symbols that refer to external realities. Therefore, new symbols are stored and processed in another realm within the self. However, the model defines the self ambiguously as the entire spectrum of semantic, perceptual contents by mental activity. Therefore, we need to improve this ambiguity to transform the IC model to an ontic (material) system model. In the following review, we use $A$ for a given sequence, instead of $M$, and the sequences $B$ and $C$, respectively, for symbols that are directly and indirectly derived from the given sequences (Sections 3 and 4 ). 


\section{The Inverse Causality}

We review the details of the logic of the IC model in this section to represent the IC process in terms of material interactions in Section 4 . We use different characters from those in the previous study (Section 2) to denote the self (subject) and derive entities' symbols for the systematic use of characters to develop the model.

\subsection{Generation of Symbols for the External Reality That Is Measured Directly (ICM)}

The IC model assumes the unique-successor principle (US principle): Any element of the sequence has a unique successor. Formally, we can define it as follows: Given a sequence of elements $(\epsilon X)$, if $x i=x j(x i, x j \in X)$, then $G(x i)=G(x j)$, where $G(x)$ is a successor of $x(\epsilon X)$. Equivalently, as its contraposition, if $G(x i) \neq G(x j)$, then $x i \neq x j$. This principle does not exclude cases where different elements are followed by the same element; $G(a)=G(b)$ when $a \neq b$, implying that the same elements can recur. The IC's logic that $\mathrm{G}(\mathrm{xi}) \neq \mathrm{G}(\mathrm{xj})$ entails $\mathrm{xi} \neq \mathrm{xj}$ is the inverse causality, equivalent to the contraposition of the unique successor principle.

For example, consider the following sequence of perceptual elements $(A)$ with the set of elements A (we use " $A$ ", instead of $M$ used in Section 2, for the systematic use for development).

$$
a 0, a 1, \ldots, a 0, a 2, \ldots
$$

This sequence violates the US principle, in which $a 0$ is followed by $a 1$ or $a 2$. According to the inverse causality, the first $a 0$ (i.e., $\left.\mathrm{G}^{-1}(a 1)\right)$ and the second $a 0$ (i.e., $\left.\mathrm{G}^{-1}(a 2)\right)$ must be different because $a 1 \neq a 2$. However, for the self, it is an undoubted fact that the first and the second $a 0$ are the same. In other words, elements in the sequence are indubitable, direct experiences for the self, with their identity given as real at a departure point of argument; they can never be differentiated into different elements [23]. According to the above rule of inverse causality, we introduce new elements or symbols in $A$ 's background. Therefore, we introduce new symbols foreign to sequence $A$, say $b 1$ and $b 2$, behind each $a 0$. We have then a new sequence as follows:

$$
(a 0, b 1), a 1, \ldots,(a 0, b 2), a 2, \ldots
$$

The IC operation on $A$ generates new symbols ( $b 1$ and $b 2$ ) that do not belong to $\mathbf{A}$. We operated the IC on $A^{\prime}$ 's state changes; designated ICM in the previous study (Section 2). Then, we have a new sequence $B$ with a set of symbols (B), and we call them the symbols for the second-order reality.

$$
\mathbf{B}=\{b 1, b 2\} .
$$

(Note: concerning Sequence $1 \mathrm{a}$, we can give the following case: $a 0, a 0, \ldots, a 0, a 1, \ldots$. This sequence also violates the US principle, in which the $a 0$ is followed by $a 0$ or $a 1$. This case also leads to the same conclusions as the above case.)

\subsection{IC Process as Measurement for External Observer}

In the IC model, i.e., an internalist, phenomenological model, the external entity $E$ exists in the form of symbols generated by an IC process within the subject. By contrast, we can take an externalist view, seeing the whole, including the subject and its external entity, from the outside. Here, the externalist stance means to assume a theoretical observer who observes the entire system, including a focal subject and other entities with which the subject interacts. The observer can be called "the meta-observer" [21,24] because it observes the system from nowhere and nowhen in the system.

From this externalist viewpoint, the IC process appears to be a process in which the subject measures the entity's different states, $e 0$ and $e 1$. Here, the subject discriminates between the different states, changing to $a 1$ or $a 2$ (Sequence 1b). Discrimination conceptually corresponds to causation between two entities [24]. The entity causes the subject's response, in the sense that differences in the entity's state are followed by differential 
responses by the subject. Measurement is the causative discrimination by a measurer between different states of an object $[25,26]$. Therefore, the IC process is equivalent to the process of measurement for an external observer.

\subsection{Generation of Symbols for the External Reality That Is Measured Indirectly (ICW)}

Let us introduce the ICW process. Consider the following sequence of perceptual elements $(A)$ with the set of elements $\mathbf{A}$ :

$$
a 0, a 0, a 1, \ldots, a 0, a 0, a 2, \ldots
$$

This sequence violates the US principle because the second $a 0$ is followed by $a 1$, whereas the fourth a 0 by $a 2$, as in Sequence $1 b$. By operating IC on the sequence, we have

$$
a 0,(a 0, b 1), a 1, \ldots, a 0,(a 0, b 2), a 2, \ldots
$$

Here, we can identify that the first and the third $a 0$ have the same successor, $a 0$. Therefore, no IC operation is needed since it fulfills the US principle at the $A$ level. This understanding may be true if we deal with a pair of the same changes in isolation. However, if we consider that the sequence contains the three kinds of state changes from $a 0$ to $a 0, a 1$, or $a 2$. Under this context, the subject can derive an external reality's symbol for the first and third $a 0$, say $b 0$. Then, we have

$$
(a 0, b 0),(a 0, b 1), a 1, \ldots,(a 0, b 0),(a 0, b 2), a 2, \ldots
$$

Next, we can notice that Sequence 2c does not fulfill the US principle at the whole level: the first and second $(a 0, b 0)$ do not have the unique successor: They are followed by $(a 0, b 1)$ for the former case and by $(a 0, b 2)$ for the latter. Therefore, by operating IC again on Sequence $2 c$ at the whole level (ICW), we introduce new symbols, $c 1$ and $c 2$, respectively, to the first and second $(a 0, b 0)$. Then, we have

$$
(a 0, b 0, c 1),(a 0, b 1), a 1, \ldots,(a 0, b 0, c 2),(a 0, b 2), a 2, \ldots .
$$

The sequence now fulfills the US principle at the whole level. We have a new sequence $C$ with a set of symbols $(\mathbf{C})$ and call them the symbols for the third-order reality.

$$
\mathbf{C}=\{c 1, c 2\}
$$

Note that this fulfills the principle only for the operated part. In other words, the sequence can repeatedly violate the principle in the future direction. The subject realizes the third-order reality (i.e., making it real) mediated through non-unique changes of a derived sequence $B$, i.e., $b 0 \rightarrow b 1$ or $b 2$ ( $A$ is in the same state $a 0$ for either change). In this sense, deriving $c 1$ and $c 2$ is an indirect measurement for the externalist, i.e., the meta-observer's view. According to the indirectness in measurement, $C^{\prime}$ 's elements refer to the states of the hidden external reality for the subject (see Section 4.6 for details).

\section{Cognizer System Operating Inverse Causality}

\subsection{Measurement and Measurer}

The logic of the IC process, reviewed in Section 3, proceeds backward in time. Therefore, applying the IC process to living systems requires transforming the process to a material (ontic) process proceeding forward in time. For this purpose, we require a general concept of a material entity functioning as a material subject, without recourse to specific entity models, such as molecules and cells. A cognizer is a material entity model, developed to explore the probability of events in living systems [21,24,27].

A cognizer takes a specific state every moment in time and changes the state in relation to others. This related state-change is called "cognition", which includes any material state-changes, physical or biological, including cognition in cognitive and brain sciences as 
a particular case. Each cognizer $(C i)$ is identified with a pair of its motion function $f c i$ and the state set or space $(\mathbf{C i})$, i.e., $(f c i, \mathbf{C i})$. Any system composed of cognizers in intercognition (or interaction) is called a cognizers system [21,24,27].

A "cognizers" system (" $\mathrm{s}$ " for cognizer) can be hierarchically organized in a nested fashion, where the parts constitute a whole synchronically or diachronically [28]. A particular single cognizer can be a system of sub-cognizers, which is, therefore, called a "cognizer" system (no "s" for cognizer) when we focus on a particular cognizer as a system of subcognizers. In other words, a cognizers system is a system made of cognizers; and a cognizer (i.e., a system made of sub-cognizers) is an element of the cognizers system. In this paper, we take an internalist stance to the problem by dealing with a focal cognizer only, without considering other component cognizers that constitute the focal cognizer's environment in the cognizers system (Figure 2).

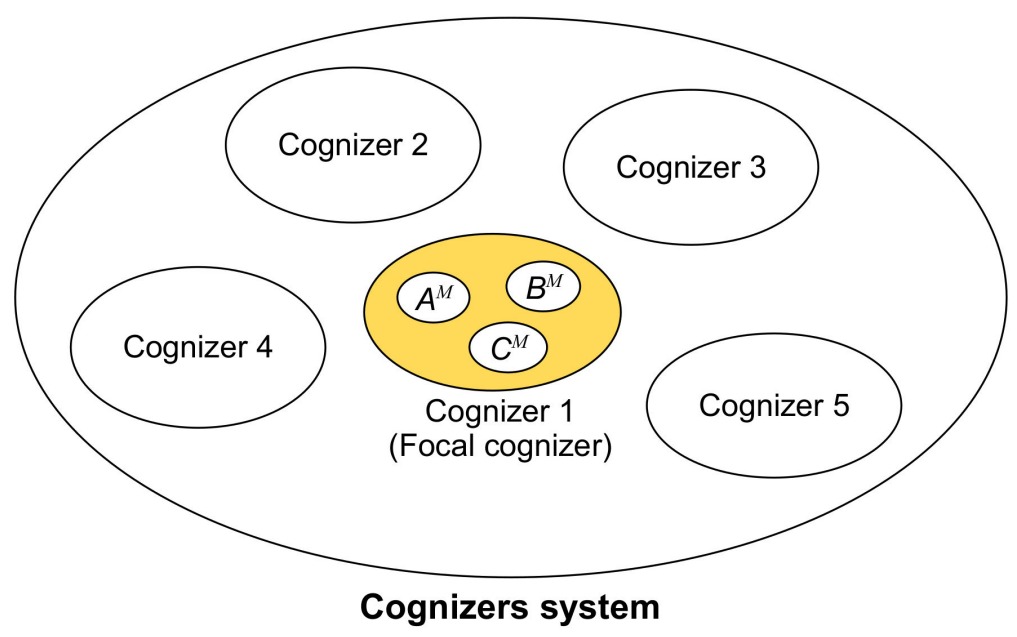

Figure 2. The cognizers-system model (CS model) assumes the whole set of cognizers in interaction, viewed from the meta-observer. The internalist, phenomenological model of this paper describes a focal cognizer (Cognizer 1: yellow circle) only, composed of cognizers at a lower level $\left(A^{M}, B^{M}\right.$, and $C^{M}$ are shown here for discussions in Section 4).

The IC model derives new symbols based on given differential state-changes from the same state (e.g., $a 0 \rightarrow a 0$ or $a 1$ ). To transform the derivation process into the cognizerssystem model (CS model), we assume a focal cognizer (i.e., a subject system) to have a sub-cognizer that can "measure" something by one-to-many state changes. Here, we introduce the concept of "measurer": Measurer is a class of cognizers with a property of returning to a baseline state under a particular environmental state after changing to different states. The baseline state is not necessarily unique but can be a plural number of states.

This concept grasps a particular property common in ubiquitous apparatuses for signal transduction or information transmissions, such as cell-surface receptors, ion channels [29], molecular switches [30], and neurons [31]. The measurement by IC differs from measurement in the sense of observation; therefore, we call the former measurement "ICmeasurement" to avoid confusion when necessary. The IC-measurement is not observation but the realization that it does not presuppose the existence of an object to be measured. It is a process of creating an object as an it-from-bit process. The IC-measurement is the derivation (generation) of symbols referring to object's states by state changes of a measurer from a baseline state.

To illustrate the IC-measurement figuratively, consider two entities: a meter and a reader, without assuming any object measured by the meter (Figure 3). Both constitute the subject; it is not that the reader is the subject who reads the meter existing externally. Suppose that the meter is set in a baseline state (0), which then changes to a particular state 1 or 2 . In a particular baseline state, the reader changes its state by reading 0 and, 
then, 1 (not shown in the figure). The reader can derive a difference between the first and the second $a 0$ (red or blue arrows) in the form of the reader's states. Those states refer to different states of something measured by the meter. The meter corresponds to $A$, while the reader corresponds to $B$ in the IC model (Section 3). With this IC-measurement, something external is embodied as the reader's state.

ao

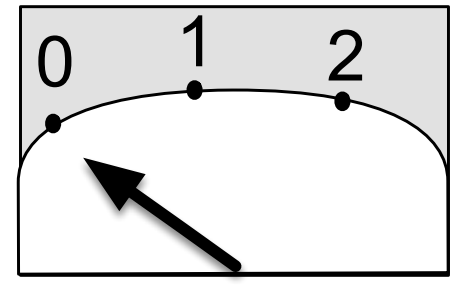

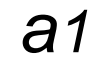
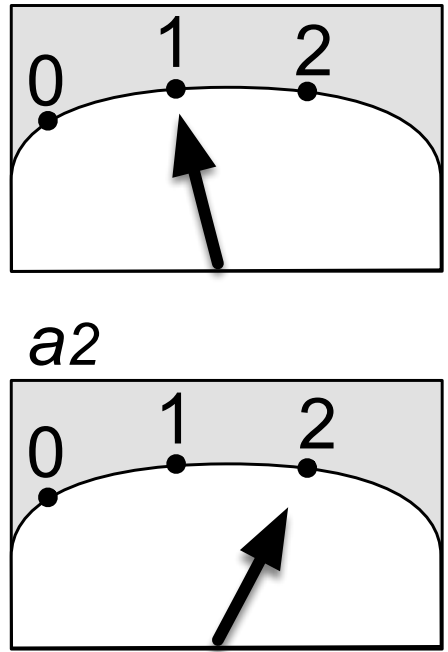

Figure 3. Measurement (or observation) as a material process proceeds from left to right, forward in time as the arrows indicate. Events occur in the order of $a 0, a 1, \ldots, a 0, a 2$ in the meters. However, the inverse causality process proceeds from right to left, backward in time by logic. New symbols, $b 1$ and $b 2$ in Sequence $1 \mathrm{~b}$ are generated behind $a 0$ from $a 1$ and $a 2$, respectively, with the inverse-causality (IC) model. The measurement based on IC requires at least two entities: a meter and its reader (not shown), both of which compose the subject, without assuming any object to be measured; the meter is within the subject, not outside.

\subsection{IC-Measurement in Cognizer: Two-Component Model}

Let us represent the IC process-in-logic (Section 3) using a cognizer system in the CS model. We suppose a focal cognizer as the subject, composed of sub-cognizers functioning as the measurers (Figure 2). Serial actions of measurement (cognitions) are modeled for the IC process to operate functionally. One measurer's state is measured by the second measurer, followed by the third measurer, and so on.

Let us begin with a simple case: a focal cognizer $S$ (Cognizer 1 in Figure 2) is composed of two measurer cognizers, $A^{M}$ (with state set $\mathbf{A}^{\mathbf{M}}$ ) and $B^{M}$ (with state set $\mathbf{B}^{\mathbf{M}}$ ). $A^{M}$ exhibits a sequence of states (i.e., sensor data), such as $a 0, a 1, \ldots$, which correspond to a sequence of $A$ used in the IC model (Section 3.1). Another measurer $\left(B^{M}\right)$ measures (reads) $A^{M \prime} \mathrm{s}$ state. The derivation of new symbols in sequence $B^{M}$ based on $A^{M}$ 's state changes can be represented as the $B^{M}$ 's generation of a state sequence by cognizing $A^{M}$. Table 1 shows a forward-in-time process within cognizer $S$ involving cognitions by measures.

Table 1. A focal cognizer $S$ is composed of two measurer cognizers, $A^{M}$ and $B^{M}$. In addition, $a 0$ and $b 0$ are baseline states for the measurers $A^{M}$ and $B^{M}$, respectively. Each state of $S$, such as $x$ and $y$, includes a cognition by the measurers. One-to-many changes are highlighted with red symbols, such as $a 0 \rightarrow a 1$ or $a 2$. The measurers change their states on a fine-grained timescale, $\mathrm{t} 0, \mathrm{t} 1, \mathrm{t} 2, \ldots, \mathrm{tn}, \mathrm{tn}+1, \mathrm{tn}+2$. The cognizer changes its states on a coarse-grained timescale, $\mathrm{T} 0, \mathrm{~T} 1, \ldots, \mathrm{TN}, \mathrm{TN}+1$.

\begin{tabular}{|c|c|c|c|c|c|c|c|c|c|c|c|c|}
\hline Measurer/Time & to & $t 1$ & $t 2$ & $t 3$ & $t 4$ & & $t n$ & $t n+1$ & $t n+2$ & $t n+3$ & $t n+4$ & \\
\hline$A^{M}$ & $a 0$ & $a 0$ & $a 1$ & $a 0$ & $?$ & . & $a 0$ & $a 0$ & $a 2$ & $a 0$ & $?$ & . \\
\hline$B^{M}$ & $b 0$ & $b 0$ & $b 0$ & $b 1$ & $b 0$ & $\ldots$ & $b 0$ & $b 0$ & $b 0$ & $b 2$ & $b 0$ & $\ldots$ \\
\hline $\begin{array}{l}\text { Cognizer } S \\
\text { state (Time) }\end{array}$ & \multicolumn{2}{|c|}{$x(T 0)$} & \multicolumn{2}{|c|}{$y(T 1)$} & & & \multicolumn{2}{|c|}{$x(T N)$} & \multicolumn{2}{|c|}{$z(T N+1)$} & & \\
\hline
\end{tabular}


In this model, each "state" of $S(x, y, z$ in Table 1$)$ includes a "cognition" by two measurers $\left(A^{M}, B^{M}\right)$; for instance, state $\mathrm{x}$ involves a lower-level cognition $(a 0, b 0) \rightarrow(a 0, b 0)$ from $\mathrm{t} 0$ to $\mathrm{t} 1 \mathrm{in}$ Table 1 . The measurers change their states on a fine-grained timescale, $\mathrm{t} 0, \mathrm{t} 1$, $\mathrm{t} 2, \ldots, \mathrm{tn}, \mathrm{tn}+1, \mathrm{tn}+2$. This modeling is introduced due to the assumption that the measurers return to a baseline state after a cognition(s), taking two time units. Therefore, a single state of $S$ includes one cognition taken by the measurers. Thus, the cognizer changes its states (i.e., cognition at the cognizer $S$ level) on a coarse-grained timescale: $x$ (T0), $y$ (T1), . , $x(\mathrm{TN}), z(\mathrm{TN}+1)$. "TN" indicates the time when the same state of a cognizer (e.g., $x$ ) recurs as that which occurs at $\mathrm{T} 0$; " $\mathrm{tn}$ " indicates the time for the measurer level that corresponds to $\mathrm{TN}$, as shown in Table 1.

As shown in the IC process from Sequences (1a,b) (Section 3.1), new symbols $b 1$ and $b 2$ are generated behind $a 0$, backward in time, based on a one-to-many correspondence, where $a 0 \rightarrow a 1$ and $a 0 \rightarrow a 2$, respectively. However, the cognizer model describes that $B^{M}$

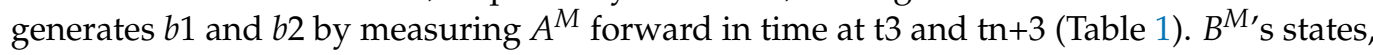
$b 1$ and $b 2$, are symbols that refer to states of something external to $S$, measured (detected) by the $A^{M}-B^{M}$ coupling (those states are supposed to occur on $\mathrm{t} 0-\mathrm{t} 1$ and on $\mathrm{tn}-\mathrm{tn}+1$ when $S$ is in state $x$ ). This process is the realization of external reality.

Living systems, such as cells, have receptor proteins on their cell membranes. The receptors, the counterpart of $A^{M}$, take different molecular structures as their states, changing by ligand binding. This event triggers the production of another type of molecule called the second messenger. For a living cell, ligand molecules or stimuli are external entities that do not belong to the cell components. What is measured by $A^{M}$ is external to $S$, whose measurement is a reliable base for a subject cognizer $S$ to distinguish the events generated by things existing outside, from the events generated only by internal processes, such as hallucinations (see Section 4.5).

Let us pay attention to the temporal framework of the model in Table 1 (also applied to Tables 2 and 3). The process of the subject cognizer $(S)$ is framed on two timescales at the level of measurers ( $t$ ) and that of the system of measurers (T). In a hierarchical system, a process at a given level proceeds at a lower rate than processes at the component level $[28,32,33]$. For example, a metabolic process of a cell can be described as changes in metabolites' concentrations as cell states, which occur at a lower rate than chemical reactions between molecules. Similarly, an animal behavior can be described as changes in bodily states, which occur at a lower rate than the rate of changes in the membrane potential of the animal's neural cells.

An internalist model focusing on a single cognizer (Cognizer 1 in Figure 2) can describe it as a system organized in a nested hierarchy with a specific timescale $(t$ or $T)$ for each level of the system. Therefore, different timescales for different levels are defined within the internalist model. In contrast, when we take an externalist stance, in which a focal subject cognizer is described in relation to other cognizers in the environment (Cognizers 2-5 in Figure 2), the externalist needs to coordinate the time occurring in different cognizers by introducing a common temporal framework in which they are situated.

\subsection{IC-Measurement in Cognizer: Three-Component Model}

In the model in Table $1, S$ is composed of $A^{M}$ and $B^{M}$ as a sensor and a signal transducer (information transmitter). Next, let us add another measurer for $B^{M}$ to represent an effector; note that effectors can be measurers if they have a baseline state. For this development, we add a third measurer that measures $B^{M}$. We could also incorporate other classes of sub-cognizers that play roles in the metabolism and memory, including measurers and non-measurers. However, we omit them to focus on the percept (cognition)-reality problem extended to living systems.

For parsimony of character use and other reasons $\left(C^{M}\right.$ is used for the ICW process in Section 4.6), let us replace $B^{M}$ with $B^{M} 1$ and add measurer $B^{M} 2$ that measures $B^{M} 1$. $B^{M} 1$ and $B^{M} 2$ have, respectively, the baseline states $b 1-0$ and $b 2-0$. Table 2 shows a process of state changes of the three measurers. The measurers change their states on a fine-grained 
timescale, $\mathrm{t} 0, \mathrm{t} 1, \mathrm{t} 2, \ldots, \mathrm{tn}, \mathrm{tn}+1, \mathrm{tn}+2$. The cognizer changes its states on a coarse-grained timescale: $x(\mathrm{~T} 0), y(\mathrm{~T} 1), \ldots, x(\mathrm{TN}), z(\mathrm{TN}+1)$. "TN" indicates the time when the same state of a cognizer (e.g., $x$ ) recurs as that occurs at T0; " $\mathrm{tn}^{\prime}$ indicates the time for the measurer level that corresponds to $\mathrm{TN}$ as shown in Table 2.

Table 2. A focal cognizer $S$ is composed of three measurer cognizers, $A^{M}, B^{M} 1$, and $B^{M}$, representing a sensor, a signal transducer, and an effector of a living system, respectively. In addition, $a 0, b 1-0$, and $b 2-0$ are baseline states of measurers $A^{M}, B^{M} 1$, and $B^{M} 2$, respectively. One-to-many changes are highlighted with red symbols. The measurers change their states on a fine-grained timescale, $\mathrm{t} 0, \mathrm{t} 1, \mathrm{t} 2, \ldots, \mathrm{tn}, \mathrm{tn}+1, \mathrm{t} \mathrm{n}+2$. The cognizer changes its states on a coarse-grained timescale, $\mathrm{T} 0$, $\mathrm{T} 1, \ldots, \mathrm{TN}, \mathrm{TN}+1$.

\begin{tabular}{|c|c|c|c|c|c|c|c|c|c|}
\hline Measurer/Time & to & $t 1$ & $t 2$ & $t 3$ & $t 4$ & $t 5$ & $t 6$ & & \\
\hline$A^{M}$ & $a 0$ & $a 0$ & $a 0$ & $a 1$ & $a 0$ & $a 0$ & $?$ & $\ldots$ & $\ldots$ \\
\hline$B^{M_{1}}$ & $b 1-0$ & $b 1-0$ & $b 1-0$ & $b 1-0$ & $b 1-1$ & $b 1-0$ & $b 1-0$ & $\ldots$ & $\ldots$ \\
\hline$B^{M_{2}}$ & $b 2-0$ & $b 2-0$ & $b 2-0$ & $b 2-0$ & $b 2-0$ & $b 2-1$ & $b 2-0$ & $\ldots$ & $\ldots$ \\
\hline $\begin{array}{l}\text { Cognizer } S \\
\text { state (Time) }\end{array}$ & & $x(T 0)$ & & & $y(T 1)$ & & & & \\
\hline (continued) & $t n$ & $t n+1$ & $t n+2$ & $t n+3$ & $t n+4$ & $t n+5$ & $t n+6$ & & \\
\hline$A^{M}$ & $a 0$ & $a 0$ & $a 0$ & $a 2$ & $a 0$ & $a 0$ & $?$ & $\ldots$ & $\ldots$ \\
\hline$B^{M_{1}}$ & $b 1-0$ & $b 1-0$ & $b 1-0$ & $b 1-0$ & $b 1-2$ & $b 1-0$ & $b 1-0$ & $\ldots$ & $\ldots$ \\
\hline$B^{M_{2}}$ & $b 2-0$ & $b 2-0$ & $b 2-0$ & $b 2-0$ & $b 2-0$ & $b 2-2$ & $b 2-0$ & $\ldots$ & $\ldots$ \\
\hline $\begin{array}{l}\text { Cognizer } S \\
\text { state (Time) }\end{array}$ & & $x(T N)$ & & & $z(T N+1)$ & & & & \\
\hline
\end{tabular}

$B^{M} 2$ discriminates between different $B^{M} 1^{\prime} s$ states, $b 1-1$ (at $\left.\mathrm{t} 4\right)$ and $b 1-2($ at $\mathrm{tn}+4)$, changing to $b 2-1$ (at $\mathrm{t} 5$ ) and $b 2-2($ at $\mathrm{tn}+5)$. This process indicates a distinction generated by $A^{M}-B^{M} 1$ coupling is conveyed to $B^{M} 2$ (effector). Each "state" of $S(x, y$, and $z)$ includes "cognitions" by three measurers, $A^{M}, B^{M} 1$, and $B^{M} 2$; for instance, "state" $x$ involves lower-level "cognitions", $(a 0, b 1-0, b 2-0) \rightarrow(a 0, b 1-0, b 2-0) \rightarrow(a 0, b 1-0, b 2-0)$, from t0 to t2. $B^{M} 2$ functions as the effector that uses symbols generated by the IC-measurement of $A^{M}-B^{M} 1$ coupling.

The IC operation-in-logic makes a perceptual sequence deterministic as described in Section 3, whereas the state sequences of cognizer $S$ in the above representation (Tables 1 and 2) do not fulfill the US principle. Their state changes are indeterministic: $(a 0, b 0) \rightarrow(\underline{a}, b 0)$ or $(\underline{a 2}, b 0)$ in Table 1 , and $(a 0, b 1-0, b 2-0) \rightarrow(\underline{a 1}, b 1-0, b 2-0)$ or $(\underline{a 2}, b 1-0$, $b 2-0)$ in Table 2 (see underlined parts). The reason for indetermination is because new IC-generated symbols come downstream (forward in time) in this model. The states $b 0$ and $b 1$ are generated, respectively, at $\mathrm{t} 3$ and $\mathrm{tn}+3$ (red symbols, marked orange) in Table 1 . Likewise, the states $b 1-1$ and $b 1-2$ are generated at $t 4$ and th +4 (red symbols, marked orange), respectively, in Table 2, although they are generated at t 0 and tn by the IC logic (Section 3).

The indeterministic changes imply that the subject cognizer is not a deterministic whole, but an open system in the sense that it is surrounded by the unknown external reality. The cognizer can generate symbols referring to states of the external reality, using its component measurers, such as $B^{M}$ or $B^{M}$. If a given cognizer is a deterministic whole, it is a closed system that cannot do the IC operation because it already fulfills the US principle.

However, a subject cognizer as an open system can manage consequent events deterministically in a sense, using IC-generated symbols by taking different cognitions (actions) to a given datum: from " $(a 0, b 1-0, b 2-0)$ " at $\mathrm{t} 2$ or $\mathrm{tn}+2$ to " $(a 0, b 1-0, \underline{b 2-1})$ " at $\mathrm{t} 5$ or " $(a 0, b 1-0$, $\underline{b 2-2})^{\prime \prime}$ at $\mathrm{tn}+5$, which affect events occurring at $\mathrm{t} 6 / \mathrm{tn}+6$ ("?" in yellow in Table 2$)$. We focus on this aspect of cognition in the next section. 


\subsection{IC-Generated Symbols for Adaptive Action}

Thus far, we have focused on the distinction ("discrimination") aspect of state changes to generate new symbols using IC operations. Another essential aspect of state change is what the CS model calls "selectivity" [24]. Selectivity is defined as a cognizer's property determining a particular state as the next among many potential states under a given environmental state to take a particular relation with the environment.

Selectivity plays a vital role in adaptive action. For example, consider a car driver observing a traffic signal, which becomes red, yellow, and green. The driver can distinguish each color from others; this is the discrimination ability of the driver. The driver has another property, which can potentially take many kinds of responses to each color: for instance, the driver could stop for green, go for red, and U-turn for yellow. This property is the "selectivity" in the driver's state changes in relation to the signal states, which affects the events that the driver will experience.

In Table $2, A^{M}, B^{M} 1$, and $B^{M} 2$ are interpreted as a sensor, a signal transducer, and an effector of a living system, respectively. Now, let us ask: What determines the sensor $\left(A^{M}\right)$ state at t6 or tn +6 (marked "?" in yellow in Table 2)? The primary answer is in how the sensor's state difference links to the effector's state changes (b2-1 or $b 2-2)$, affecting the staterelation to the unknown external entities. A "How-link" indicates the selectivity aspect of cognition, which relates the focal cognizer to the external reality as a teleonomic property.

For example, this describes how neural cells are connected with other cells and how molecular reactions are connected with other reactions. Another factor is the unknown properties of the external entity. Any cognizer cannot completely control the events to experience using the discrimination ability and selectivity. This incomplete controllability is simply because the final relation between the subject and the environment is determined by the two. For example, an organism cannot manage its fate completely by its own ability when facing a predator.

\subsection{IC Process in Bacterial Chemotaxis}

Cells can generate symbols for the external entities' states by producing molecules, called second messengers. Many bacterial species have chemotaxis for swimming toward a denser food area by sensing food molecules via receptor membrane proteins that can react to the molecules as a signal. When the flagella rotate counterclockwise (CCW), a bacterial cell swims in a smooth, directed manner. The smooth-swimming is interrupted when the flagella rotate clockwise $(\mathrm{CW})$, which causes the bacterium to tumble and change its swimming direction $[34,35]$.

Escherichia coli has five transmembrane proteins (methyl-accepting chemotaxis proteins, MCP), which are receptors for attractant and repellent chemicals [36,37]. For example, the Tar receptors bind to an amino acid (aspartate) directly. CheA, a histidine kinase, is tightly associated with and regulated by these receptors. When attractant stimuli, such as amino acids or sugars, do not bind to the receptors, their molecular configurations cause the activation of CheA, leading to the autophosphorylation of CheA. The phosphorylated CheA (denoted CheA-P), in turn, phosphorylates another kind of protein, CheY. The phosphorylated CheY (CheY-P) diffuses through the cell and encounters a flagellar motor. The CheY-P binds to a flagellar-motor-switch protein called FliM, making the rotation clockwise and the bacterium tumble [35].

In contrast, when the amino acids bind to receptors as stimuli, the receptors change the molecular configuration, which does not activate CheA. The resultant decreases in CheA-P then decrease the concentration of CheY-P (i.e., an activated state). As a result, the flagellar motor rotates counterclockwise, making the bacteria swim smoothly. As a consequence, bacterial cells swim toward attractants or away from repellent molecules.

A baseline state of the receptor (MCP) can change by methylation from another protein (CheR). In other words, the methylated receptor (in another baseline state) reduces the affinity to bind stimulus molecules and, hence, increases the activation rate of CheA, leading to an increase in phosphorylated CheY, thereby making the flagellate tumble. This 
modification of the receptor molecules functions as an adaptation of the bacterial cell to a given stimulus concentration.

When the concentration of the stimulus becomes higher, the sensor now cannot activate CheA. This event leads to a decrease in CheY-P, making the bacteria swim in a smooth straight direction. In the chemotaxis system, the degree of methylation is a type of memory stored in the receptor's molecular state. (Another protein CheB, in a phosphorylated (activated) state, can remove the methyl from methylated MCPs. However, the decrease in CheA-P by stimulus chemicals binding to receptors reduces the activated $\mathrm{CheB}$, which then decreases the demethylation rate of $\mathrm{MCP}$ ). With this methylation system, bacterial cells can detect changes in the stimulus concentration in their environments [35].

We can interpret that these receptors and signaling molecules are the measurers in that they have activated states, returnable to an inactivated, baseline state. Here, the receptor protein (MCP) with CheA can correspond to $A^{M}$, CheY to $B^{M} 1$, and $B^{M} 2$ to the switch-proteinassociated flagellar motor in Table 2. In the IC process using the CS model, the measurers may have two more baseline states. By shifting to another baseline state, the measurers can change the measurement mode with different affinities, like methylated MCPs.

Lastly, imagine a bacterial mutant with a modified structure of signal receptors (MCP) that reacts to certain metabolites excreted through its membrane, instead of signal ligands coming from the environment; or a mutant producing a mutated CheY protein that can be phosphorylated (activated) by metabolites other than CheA-P. They may cause maladaptive flagellar mortar regulation for swimming, which may be considered a kind of hallucination in a living cell, similar to those in animals. During the long period of life evolution, there would likely occur many kinds of mutants that suffered from disorders causing inappropriate distinctions between entities of the two realms (external or internal origins).

\subsection{ICW Process in Cognizer}

As discussed in Section 4.4, a focal cognizer, the subject, can manage consequent events occurring to the cognizer by taking different cognitions (actions) for a given sensor datum. However, if a given cognition is repeated more than two times in the long run, the cognizer may not necessarily obtain the same sensor data as a result. For example, consider a game in which a player draws one card from many lying on a table face down after shuffling. When the player repeats the game, he finds the same visual data about cards lying on a table face down after shuffling. Here, he takes a particular action of taking one card for each trial, but the results can vary for repeated trials depending on the cards' configurations.

Therefore, the same action against different card configurations will result in different events occurring to the player. This kind of uncertainty in events occurring after a particular action (cognition) is due to non-discriminability among different configurations (states) of the environment. Consider another case where the cards are face up. The player can now take the same or different cards with certainty, depending on his discriminative selection or preference. This uncertainty originates from the different states of a hidden reality that can be derived by the IC operation on the whole sequence (ICW; Sequences $(2 \mathrm{a}-\mathrm{c})$ in Section 3.3). Here, "hidden" indicates a state or condition that cannot be measured by the $A^{M}-B^{M}$ coupling, i.e., ICM (Sections 4.2 and 4.3).

We can represent this IC process-in-logic using a cognizer system in the CS model as shown in Table 3. Here, a focal cognizer is composed of the measurers, $A^{M}, B^{M}$, and $C^{M}$, i.e., a sensor, a signal transducer, and a hypothetical apparatus of the ICW operation to detect a hidden reality, respectively. Here, we omit the effector for simplicity in representation. Table 3 modifies Table 2 by adding the first block of state $\mathrm{x}$ of cognizer $\mathrm{S}$ and replacing $B^{M} 2$ with $C^{M}$. The measurers change their states on a fine-grained timescale, $t 0$, $\mathrm{t} 1, \mathrm{t} 3, \ldots, \mathrm{tn}, \mathrm{tn}+1, \mathrm{tn}+2$. The cognizer changes its states on a coarse-grained timescale: $x$ (T0), $x(\mathrm{~T} 1), y(\mathrm{~T} 2), \ldots, x(\mathrm{TN}), x(\mathrm{TN}+1), z(\mathrm{XN}+2)$. "TN" indicates the time when the same state change of a cognizer (e.g., $x \rightarrow x$ ) recurs as that occurs on $\mathrm{T} 0-\mathrm{T} 1$; " $\mathrm{tn}^{\prime \prime}$ indicates the time for the measurer level that corresponds to TN as shown in Table 3. 
Table 3. A focal cognizer $S$ is composed of three measurer cognizers, $A^{M}, B^{M}$, and $C^{M}$, representing a sensor, a signal transducer, and a hypothetical apparatus of the IC operation on the whole sequence (ICW) of a living system, respectively. In addition, $a 0, b 1-0$, and $b 2-0$ are the baseline states of the measurers $A^{M}, B^{M} 1$, and $B^{M} 2$. One-to-many changes are highlighted with red symbols. The measurers change their states on a fine-grained timescale, $t 0, t 1, t 3, \ldots, t n, t n+1, t n+2$. The cognizer changes its states on a coarse-grained timescale, $\mathrm{T} 0, \mathrm{~T} 1, \ldots, \mathrm{TN}, \mathrm{TN}+1$.

\begin{tabular}{|c|c|c|c|c|c|c|c|c|c|c|c|c|}
\hline Measurer/Time & to & $t 1$ & $t 2$ & $t 3$ & $t 4$ & $t 5$ & $t 6$ & $t 7$ & $t 8$ & $t 9$ & & \\
\hline$A^{M}$ & $a 0$ & $a 0$ & $a 0$ & $a 0$ & $a 0$ & $a 0$ & $a 1$ & $a 0$ & $a 0$ & $?$ & $\ldots$ & $\ldots$ \\
\hline$B^{M}$ & $b 0$ & $b 0$ & $b 0$ & $b 0$ & $b 0$ & $b 0$ & $b 0$ & $b 1$ & $b 0$ & $b 0$ & $\ldots$ & $\ldots$ \\
\hline$C^{M}$ & $c 0$ & $c 0$ & $c 0$ & $c 0$ & $c 0$ & $c 0$ & $c 0$ & $c 0$ & $c 1$ & $c 0$ & $\ldots$ & $\ldots$ \\
\hline $\begin{array}{l}\text { Cognizer } S \\
\text { state (Time) }\end{array}$ & & $x(T 0)$ & & & $x(T 1)$ & & & $y(T 2)$ & & & & \\
\hline (continued) & $t n$ & $t n+1$ & $t n+2$ & $t n+3$ & $t n+4$ & $t n+5$ & $t n+6$ & $t n+7$ & $t n+8$ & $t n+9$ & & \\
\hline$A^{M}$ & $a 0$ & $a 0$ & $a 0$ & $a 0$ & $a 0$ & $a 0$ & $a 2$ & $a 0$ & $a 0$ & $?$ & $\ldots$ & $\ldots$ \\
\hline$B^{M}$ & $b 0$ & $b 0$ & $b 0$ & $b 0$ & $b 0$ & $b 0$ & $b 0$ & $b 2$ & $b 0$ & $b 0$ & $\ldots$ & $\ldots$ \\
\hline$C^{M}$ & $c 0$ & $c 0$ & $c 0$ & $c 0$ & $c 0$ & $c 0$ & $c 0$ & $c 0$ & $c 2$ & $c 0$ & $\ldots$ & $\ldots$ \\
\hline $\begin{array}{l}\text { Cognizer } S \\
\text { state (Time) }\end{array}$ & & $x(T N)$ & & & $x(T N+1)$ & & & $z(T N+2)$ & & & & \\
\hline
\end{tabular}

$S^{\prime}$ 's state changes (cognitions at the cognizer level) are " $x \rightarrow y^{\prime \prime}$ (T1-T2) and " $x \rightarrow z$ " (TN+1-TN+2). Using $B^{M \prime}$ s non-unique state changes " $b 0 \rightarrow \underline{b 1}$ " (t4-t7) and " $b 0 \rightarrow \underline{b 2} "$ $(\mathrm{tn}+4-\mathrm{tn}+7)$, the cognizer $S$ measures the external reality at $\mathrm{T} 1$ and $\mathrm{TN}+1$, symbolized as $b 1$ and $b 2$, which corresponds to the process from Sequences $(2 \mathrm{a}-\mathrm{c})$ in the IC model (Section 3.3). As a result, the subject generates symbols $b 1$ and $b 2$, referring to different external states, which may be used for the effector's differential actions (cognitions) to relate the subject to the environment, as discussed in Sections 4.3-4.5.

Next, we focus on the same state changes (cognitions) " $x \rightarrow x^{\prime}$ " taken by the subject on two occasions, $\mathrm{T} 0-\mathrm{T} 1$ and $\mathrm{TN}-\mathrm{TN}+1$, but followed by different events: $y$ at $\mathrm{T} 2$ and $z$ at $\mathrm{TN}+2$. The first cognition is followed by sensor datum " $a 1$ " (at t6 on T2), whereas the second cognition is followed by " $a 2$ " (at $\mathrm{tn}+6$ on $\mathrm{TN}+2$ ). This one-to-many correspondence is a chance event resulting from the non-discrimination between different states of the external reality, whose differences are hidden to the subject at $\mathrm{t} 0 / \mathrm{tn}$ (on T0/TN). According to the logic of ICW, symbols for the hidden reality can be derived from the symbols of $B$ (Section 3.3). Therefore, the third measurer, $C^{M}$, measures the difference to generate new symbols, $c 1$ and $c 2$, by discriminating between $b 1$ and $b 2$ on $\mathrm{t} 7-\mathrm{t} 8$ and $\mathrm{tn}+7-\mathrm{tn}+8$

However, according to the ICW logic, these newly derived symbols embodied as the states of $C^{M}$ are supposed to refer to the hidden reality states occurring on T0 and $\mathrm{TN}$, which are backward-in-time references. This is hindsight. For living systems, such a hindsight derivation of hidden reality is too late to avoid chance events. How can the subject use what $C^{M}$ measures to regulate its effector for living processes?

One possible answer comes from the externalist view. Living systems need not discriminate every potential difference from others occurring in the environment. They need to discriminate only the external states that are relevant to their survival/reproduction. What should be discriminated can be classified into two classes: the first is the conditions (the environmental states) that directly affect the system's activity, including physical, chemical, and biological states, and the second is the states that correlate with the first ones.

Living systems often use the second ones as signs for things that directly affect their living, such as food and natural enemies. Suppose that $A^{M}$ measures the states of a sign for something dangerous for a focal subject organism, for instance, a predator. The events occur for an external observer as follows (arrows indicates the temporal order): a predator comes near (T0) $\rightarrow$ the surrounding air vibrates, making a particular sound (T1) $\rightarrow$ the organism hears the sound (T2). 
$A^{M}$ (the organism's sensor) measures the sound on $\mathrm{T} 2, B^{M}$ (signal transducer) measures $A^{M}$, making a symbol (b1) referring to the sound state on T2, and $C^{M}$ (hypothetical apparatus) measures $B^{M}$, making a symbol (c1) referring to the dangerous state on T2. $C^{M}$ is not a transducer of the sound symbols $B^{M}$ makes but an interpreter of them. In this case, the organism made the IC-measurement of the hidden reality, a predator, mediated through measuring the air states. If symbol $c 1$ is used to link the effector appropriately (not shown in Table 3), the organism could escape the predator in time. Here, the symbol has a particular meaning, a "danger". The uncertainty regarding the surrounding air does not matter to the subject, for whom hidden danger does matter. Similarly, visual images of something are signs of an object (e.g., a predator). The eyes measure the surrounding photon field state, not the object. "A predator" is an interpretation of a given state of the photon field; different things might exist behind the visual scene.

In the above event line, let us replace an organism with a quantum physicist, the air with an experimental measurement apparatus, and a predator with the quantum reality. In this experiment, the physicist measures the apparatus states, not the hidden quantum reality, just as the organism measures the air states. From a realist stance, the apparatus is supposed to measure the quantum reality (hidden to the physicist) just as the air measures the predator (hidden to the organism). Wheeler's "it" is derived from the "apparatuselicited" answer to yes or no questions (Section 1) [17]. The symbols referring to the quantum-reality states ( $c 1$ and $c 2$ in Table 3 ) can be derived by ICW, the contraposition of the US principle (Section 3.1). It is enigmatic that a symbol for a quantum reality ("it") is detected using the US principle at the whole level (ICW), which is equivalent to the determinist principle [38] (Section 3.4).

Finally, $C^{M}$ may play a role in reducing the uncertainty of events by discovering a particular subset of sensor data (not $A^{M}$ in Table 3) unused for the IC-measurement processing by the subject but that highly correlates with $C^{M}$ 's state. This process may relate to learning. By using such data, the subject can reduce the uncertainty in events occurring at $\mathrm{t} 6 / \mathrm{tn}+6$. This process is the counterpart of seeking hidden parameters to develop a deterministic theory in quantum physics.

The search for sensor data to reduce the uncertainty of events may closely relate to one aspect of active inference proposed by Friston [3-5] in brain science, suggesting that the brain samples sensory data (signals) selectively so to minimize uncertainty (entropy) in a posterior probability distribution. However, controversy remains on the generality of this principle [39]. Fiorillo [40] points out that, in animals, the "gaze is directed not to the static and predictable parts of a visual scene but to the dynamic and uncertain parts. Exploration provides the brain with new information from its environment." His point closely concerns the previous argument that living systems use signs for reducing the uncertainty of correlated events that can directly affect their survival and reproduction. The uncertainty of sensory data about the signs does not appear to be harmful to living systems.

\section{Conclusions}

Based on the internalist, phenomenological stance, inverse causality plays an essential role in generating symbols referring to states of the external world, embodied as the states of internal components. In this sense, living systems can escape solipsism. IC operates on one-to-many state changes, which requires the system to have the machinery for repeatedly returning to an original state. Any living system as an entire system will not return to the same state frequently, because this includes subsystems of metabolic processes and memory storage. However, living systems invented a particular class of organic devices, called "measurers", and evolved them.

Living systems contain these measurers extensively, such as cell-surface receptors, ion channels [29], molecular switches in signal transduction within a cell and between cells [30], and neural cells in multicellular organisms [31]. These organic devices perform the ICmeasurements, i.e., the generation of symbols referring to the external reality, transmitted 
to effectors with interpretation. The IC-measurement underlies the fundamental element of the information processing in living systems to survive and reproduce.

To conclude, let us ask: Are living systems solipsist, positivist, or realist? Here, we can characterize the major aspects of the three models that living systems can have:

(1) A solipsist system accepts the first-order reality only (i.e., perceptual sequence $A$; Section 2), where no IC operation occurs. This system lives based on a solipsistic world model that does not distinguish between internal and external things.

(2) A positivist system accepts the first- and second-order reality ( $A$ and $B)$, where only the ICM operation occurs (Section 3.1). This system lives based on a positivist world model and only accepts external entities that can be directly measured. This system has a class of measurers that generates symbols that are foreign to the subject $\left(A^{M}-B^{M}\right.$ coupling, Section 4.2), which play an essential role in escaping a solipsist world to a positivist world. The system can distinguish the events caused by the external reality and the events caused by an internal process, such as hallucinations.

(3) A realist system accepts the first-, second-, and third-order reality $(A, B$, and $C)$, where ICW operations occur (Section 3.3). This system lives based on a realist world model, which accepts external entities that can be directly or indirectly measured. The measurers $\left(C^{M}\right)$ that measure $B^{M}\left(B^{M}-C^{M}\right.$ coupling, Section 4.6$)$ play an essential role in escaping a positivist world to a realist world. This system has the potential to reduce the uncertainty of events occurring to it by using signs for things that cannot be directly measured.

We do not necessarily need to decide on one answer among the three, because the answer may vary depending on the evolutionary stages of living systems emerging from a non-living background. In this paper, we focused on living systems that have already evolved the measurers, and we ignored their evolution and metabolic systems. Matsuno [41,42] explored the early stages of metabolic cycles in chemical and biological evolution from an internalist framework (Note that "measurement" is used as the IC-measurement in this paper, which is not identical to the measurement concept that Matsuno uses). As a perspective, the above three models might relate to evolutionary stages of the organization of living systems, including metabolic processes originating from the scene of chemical evolution, which would require future investigation.

Funding: This research received no external funding.

Institutional Review Board Statement: Not applicable.

Informed Consent Statement: Not applicable.

Acknowledgments: The author appreciates the valuable comments from the two anonymous reviewers on the previous version of this paper. One reviewer suggested to clarify the temporal framework for the internalist model of a cognizer at the levels of the measurers and the cognizer. Another reviewer suggested certain ambiguous representations and relations to works relevant to the arguments in this paper. They helped to improve and make this paper clearer.

Conflicts of Interest: The author declares no conflict of interest.

\section{References}

1. Knill, D.C.; Pouget, A. The Bayesian brain: The role of uncertainty in neural coding and computation. Trends Neurosci. 2004, 27, 712-719. [CrossRef]

2. Griffiths, T.L.; Kemp, C.; Tenenbaum, J.B. Bayesian Models of Cognition; Carnegie Mellon University: Pittsburgh, PA, USA, 2008; pp. $1-49$.

3. Friston, K.J.; Daunizeau, J.; Kiebel, S.J. Reinforcement learning or active inference? PLoS ONE 2009, 4. [CrossRef]

4. Friston, K. The free-energy principle: A unified brain theory? Nat. Rev. Neurosci. 2010, 11, 127-138. [CrossRef]

5. Seth, A.K. The cybernetic bayesian brain: From interoceptive inference to sensorimotor contingencies. Open Mind 2014, 35 . [CrossRef]

6. Von Uexküll, J. Theoretical Biology; Harcourt, Brace: New York, NY, USA, 1926.

7. Von Uexküll, J. A Foray into the Worlds of Animals and Humans: With a Theory of Meaning; University of Minnesota Press: Minneapolis, MN, USA, 2010. 
8. Sjolander, S. On the evolution of reality-Some biological prerequisites and evolutionary stages. J. Theor. Biol. 1997, 187, 595-600. [CrossRef]

9. Husserl, E. Ideas Pertaining to a Pure Phenomenology and to a Phenomenological Philosophy; First Book, General Introduction to a Pure Phenomenology; Springer: Berlin, Germany, 1983.

10. Husserl, E. Cartesian Meditations; Springer: Berlin, Germany, 1931.

11. Merleau-Ponty, M. Phenomenology of Perception; Routledge: London, UK; New York, NY, USA, 2005.

12. Zahavi, D. Husserl's noema and the internalism-externalism debate. Inquiry 2004, 47, 42-66. [CrossRef]

13. Zahavi, D. Internalism, externalism, and transcendental idealism. Synthese 2008, 160, 355-374. [CrossRef]

14. Maturana, H.R.; Varela, F.J. Autopoiesis and Cognition-The Realization of the Living; Reidel: Dodrecht, The Netherlands, 1980; ISBN 9-0277-1015-5.

15. Schrödinger, E. Nature and The Greeks; Cambridge University Press: New York, NY, USA, 1954.

16. Schrödinger, E. Mind and Matter; Cambridge University Press: New York, NY, USA, 1959.

17. Wheeler, J.A. Information, physics, quantum: The search for links. In Proceedings of the 3rd International Symposium on Foundations of Quantum Mechanics, Tokyo, Japan, 28-31 August 1989; (reproduced In Feynman and Computation). Hey, A.J.G., Ed.; Perseus Books: Cambridge, UK, 1999; pp. 309-336, ISBN 0-7382-0057-3.

18. Rössler, O. Endophysics; World Scientific Pub. Co. Inc.: Singapore, 1998; ISBN 981-02-2752-3.

19. Rössler, O. Endophysics: Descartes taken seriously. In Inside Versus Outside; Atmanspacher, H., Dalenoort, G.D., Eds.; Springer: Berlin, Germany, 1993; pp. 153-161.

20. Salthe, S.N. Theoretical biology as an anticipatory text: The relevance of Uexküll to current issues in evolutionary systems. Semiotica 2001, 134, 1-22. [CrossRef]

21. Nakajima, T. Unification of Epistemic and Ontic Concepts of Information, Probability, and Entropy, Using Cognizers-System Model. Entropy 2019, 21, 216. [CrossRef]

22. Shannon, C.E.; Weaver, W. The Mathematical Theory of Communication; University of Illinois Press: Champaign, IL, USA, 1949.

23. Nakajima, T. Is the world deterministic? Mental algorithmic process generating external reality by inverse causality. Int. J. Gen. Syst. 2001, 30, 681-702. [CrossRef]

24. Nakajima, T. Biological probability: Cognitive processes of generating probabilities of events in biological systems. J. Theor. Biol. 1999, 200, 77-95. [CrossRef] [PubMed]

25. Rosen, R. Observation and biological systems. Bull. Math. Biol. 1977, 39, 663-678. [CrossRef]

26. Rosen, R. Fundamentals of Measurement and Representation of Natural Systems; Elsevier North-Holland, Inc.: New York, NY, USA, 1978.

27. Nakajima, T. Probability in biology: Overview of a comprehensive theory of probability in living systems. Prog. Biophys. Mol. Biol. 2013, 113, 67-79. [CrossRef]

28. Nakajima, T. Synchronic and diachronic hierarchies of living systems. Int. J. Gen. Syst. 2004, 33, 505-526. [CrossRef]

29. Kadir, L.A.; Stacey, M.; Barrett-Jolley, R. Emerging roles of the membrane potential: Action beyond the action potential. Front. Physiol. 2018, 9, 1-10. [CrossRef]

30. Hörner, M.; Weber, W. Molecular switches in animal cells. FEBS Lett. 2012, 586, 2084-2096. [CrossRef]

31. Kandel, E.R.; Schwartz, J.H.; Jessell, T.M.; Siegelbaum, S.A.; Hudspeth, A.J.; Mack, S. Principles of Neural Science, 5th ed.; McGraw-Hill Professional: New York, NY, USA, 2012.

32. Simon, H. The Sciences of the Artificial, 3rd ed.; The MIT Press: Cambridge, MA, USA, 1996.

33. O'Neill, R.V.; Deangelis, D.L.; Waide, J.B.; Allen, T.F.H. A Hierarchical Concept of Ecosystems; Princeton University Press: Princeton, NJ, USA, 1986.

34. Silverman, M.; Simon, M. Flagellar rotation and the mechanism of bacterial motility. Nature 1974, 249, 73-74. [CrossRef] [PubMed]

35. Boyd, A.; Simon, M. Bacterial chemotaxis. Annu. Rev. Physiol. 1982, 44, 501-517. [CrossRef]

36. Falke, J.J.; Bass, R.B.; Butler, S.L.; Chervitz, S.A.; Danielson, M.A. The two-component signaling pathway of bacterial chemotaxis: A molecular view of signal transduction by receptors, kinases, and adaptation enzymes. Annu. Rev. Cell Dev. Biol. 1997, 13, 457-512. [CrossRef]

37. Falke, J.J.; Hazelbauer, G.L. Transmembrane signaling in bacterial chemoreceptors. Trends Biochem. Sci. 2001, 26, 257-265. [CrossRef]

38. Nakajima, T. Biologically inspired information theory: Adaptation through construction of external reality models by living systems. Prog. Biophys. Mol. Biol. 2015, 119, 634-648. [CrossRef] [PubMed]

39. Friston, K.; Thornton, C.; Clark, A. Free-energy minimization and the dark-room problem. Front. Psychol. 2012, 3, 3389. [CrossRef]

40. Fiorillo, C.D. A neurocentric approach to Bayesian inference. Nature Reviews. Neuroscience 2010, 11, 605. [CrossRef] [PubMed]

41. Matsuno, K. From quantum measurement to biology via retrocausality. Prog. Biophys. Mol. Biol. 2017, 131, 131-140. [CrossRef] [PubMed]

42. Matsuno, K. Accommodating Probability to Durability as Facing the Onset of Biological Phenomena from Within. Philosophies 2020, 5, 47. [CrossRef] 